\title{
RESULTS FROM BEAM TESTS OF UA1 U/TMP CALORIMETER MODULES
}

\author{
Tejinder S. Virdee \\ CERN, Geneva and Imperial College, London.
}

UA1 Collaboration

Aachen - Amsterdam (NIKHEF) - Annecy (LAPP) - Birmingham - Boston - CERN -Helsinki - Kiel- Imperial College, London - Queen Mary and Westfield College, London - Madrid (CIEMAT) - MIT - Padua - Paris (College de France) Rome - Rutherford Appleton Lab. -Saclay (CEN) - UCLA - Vienna Collaboration

\section{ABSTRACT}

We present results from three types of Uranium/TMP calorimeter modules constructed by the UAl Collaboration. Electromagnetic and hadronic energy resolutions have been measured using electron and pion beams in the momentum range $1 \mathrm{GeV} / \mathrm{c}$ to $70 \mathrm{GeV} / \mathrm{c}$. Results on energy linearity and spatial uniformity of response are also reported. The electromagnetic shower position resolution in the fine sampling modules has been measured using a position detector placed at a depth of $3.4 X_{0}$. The ratio of the electron to pion response has been measured both as a function of the energy and of the electric field. The high lateral and longitudinal granularity of one of the modules which includes a position detector has been used to determine the electron-pion separation as a function of energy.

\section{INTRODUCTION}

Sampling calorimeters using liquid filled ionization chambers as detection elements have several important advantages. The absence of internal amplification of charge results in a stable calibration over long periods of time provided the purity of the liquid is maintained at a sufficiently high level. There is also considerable flexibility in the size and shape of the charge collecting electrodes allowing a high granularity both longitudinally and laterally.

The motivation for the use of the room temperature liquid tetramethylpentane (TMP) for the active medium is discussed in ref. 1 . The main problem in the construction of ionization chambers, filled with room temperature liquids, is the difficulty in achieving and maintaining the required level of purity of the liquid. The chambers have to be extremely clean and leak tight and are made entirely out of stainless steel. The skins, each $0.15 \mathrm{~mm}$ thick, are laser welded on a frame of cross-section $3 \times 3 \mathrm{~mm}^{2}$. Eight electrodes, each of size $\sim(11 \mathrm{to15}) \times 13 \mathrm{~cm}^{2}$ and thickness $0.5 \mathrm{~mm}$, are held in the middle of the chamber by ceramic spacers resulting in a liquid gap of $1.25 \mathrm{~mm}$ on either side of the central electrode. The structure of a Super-gondola (SG) module is described in reference [4]. A fine grained position detector (PD) [5], with two planes of orthogonally arranged strips (pitch of $9.1 \mathrm{~mm}$ ), is placed between the first two samplings. Table 1 gives the details of the modules that are relevant to the discussion of the results presented here. The design and the results from the very forward (VFW) and forward (FW) U/TMP modules have been published in Refs. 2 and 3 respectively.
Table 1. Parameters of the U/TMP and C Modules.

\begin{tabular}{|c|c|c|c|}
\hline $\begin{array}{l}\text { Sampling } \\
\text { Number }\end{array}$ & $\begin{array}{c}\text { U Thickness } \\
(\mathrm{mm})\end{array}$ & $\begin{array}{l}\text { \# of TMP } \\
\text { Chambers }\end{array}$ & $\begin{array}{l}\text { Depth } \\
\mathrm{X}_{\mathrm{o}} \text { or } \lambda\end{array}$ \\
\hline \multicolumn{2}{|c|}{ Super-gondola (SG) } & \multicolumn{2}{|c|}{$(23.4-31.8) \mathrm{cm} \times 110 \mathrm{~cm}$} \\
\hline \multicolumn{4}{|c|}{ Position Detector at $3.4 \mathrm{X}_{\mathrm{O}}$} \\
\hline 3 & 2 & 15 & $10.1 X_{0}$ \\
\hline 4 & 2 & 10 & $6.7 \mathrm{x}_{0}$ \\
\hline $\begin{array}{l}\text { Total e.m. } \\
\text { had. } 5 \\
\begin{array}{c}6 \\
\text { Total e.m. }\end{array}\end{array}$ & $\begin{array}{r}5 \\
5\end{array}$ & $\begin{array}{l}\left(25.9 X_{0}\right. \\
12 \\
12 \\
(2.35 \lambda)\end{array}$ & $\begin{array}{l}1.03 \lambda) \\
0.66 \lambda \\
0.66 \lambda\end{array}$ \\
\hline Forward (1 & $\begin{array}{l}\text { Half Oct: } \\
10\end{array}$ & $\begin{array}{l}\text { onal Shape [ } \\
5\end{array}$ & $\begin{array}{l}\text { f. ref. 3] } \\
15 \mathrm{X}_{0}\end{array}$ \\
\hline $\begin{array}{r}\text { Very Forw: } \\
1-5\end{array}$ & $\begin{array}{c}\operatorname{ard}(\mathbf{V F W}) \\
10\end{array}$ & $\begin{array}{l}(23.4 \mathrm{~cm} \mathrm{x} \\
5\end{array}$ & $\begin{array}{l}5.1 \mathrm{~cm}) \\
0.6 \lambda\end{array}$ \\
\hline $\begin{array}{c}\text { C Module } \\
1 \\
2\end{array}$ & $\begin{array}{l}8 \times(5 \mathrm{~cm} \mathrm{Fe}+ \\
7 \times(5 \mathrm{~cm} \mathrm{Fe}+\end{array}$ & $\begin{array}{l}\text { cm scint.) } \\
\text { cm scint.) }\end{array}$ & $\begin{array}{l}2.50 \lambda \\
2.19 \lambda\end{array}$ \\
\hline
\end{tabular}

\section{RESULTS}

\section{The Response to Muons in SG Module}

Defining the signal to noise ratio as $S / N=$ (peak of the pedestal subtracted signal, summed over all six samplings, from $70 \mathrm{GeV} / \mathrm{c}$ muons / sigma of the pedestal) gives $\mathrm{S} / \mathrm{N}$ $\sim 4.4$. The mean value of the signal distribution is $\sim 60 \%$ higher than the peak value. The externally applied electric field was $12 \mathrm{kV} / \mathrm{cm}$. Similarly, in the PD we find $\mathrm{S} / \mathrm{N} \sim$ 2.0 for the short strips, i.e. in $2.5 \mathrm{~mm}$ of TMP. 


\section{The Response to Electrons}

A single tower is laterally and longitudinally large enough to contain high energy electromagnetic showers. The reconstructed electron energy, $\mathrm{E}_{\mathrm{Tec}}$, is given by $\mathrm{E}_{\mathrm{rec}}(\mathrm{GeV})=\alpha \sum_{\mathrm{i}=1,4} \mathrm{Q}_{\mathrm{i}}(\mathrm{fC})$

where $\alpha$ is asumed to be an energy independent calibration factor. The result of the fit to the measured energy resolution, with the noise $(\sim 100 \mathrm{MeV})$ subtracted quadratically, is shown in Fig. 1 and yields $\sigma(\mathrm{E}) /<\mathrm{E}\rangle=(0.120 \pm 0.004) / \mathrm{VE} \oplus(0.009 \pm 0.001)$ where $\mathrm{E}$ is in $\mathrm{GeV}$. The constant term is due to residual beam energy mismeasurement, sampling to sampling intercalibration uncertainty, longitudinal energy leakage and other smaller effects.

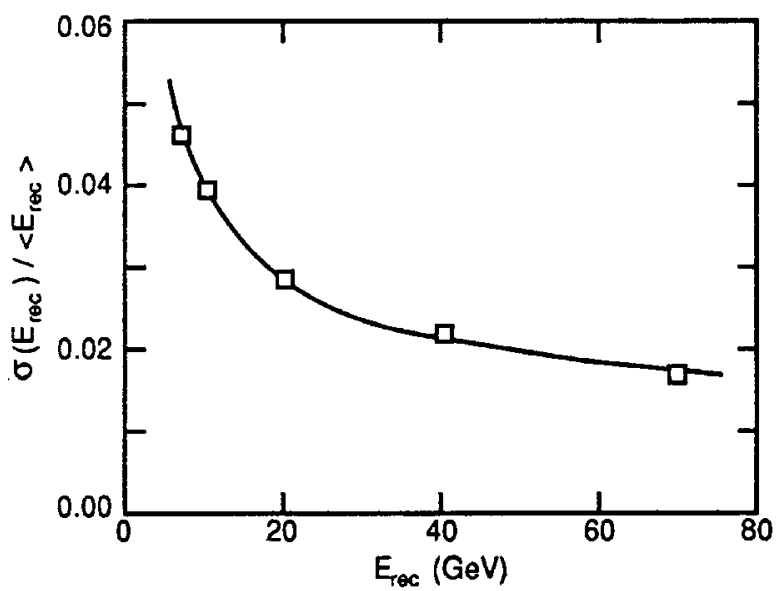

Fig.1 The energy resolution for electrons in a SG module as a function of energy.

The energy linearity is found to be better than $1 \%$ and is limited by the lack of knowledge of the exact beam energy. The electron energy resolution in the FW and VFW modules is found to be $\sigma(\mathrm{E}) /<\mathrm{E}>\sim 30 \% / \sqrt{ } \mathrm{E}$ as expected for the $10 \mathrm{~mm}$ thick $U$ absorber plates.

\section{The Response to Pions}

One SG module is not large enough to fully contain hadronic showers but the longitudinal energy leakage, and some of the lateral leakage, was captured by the $\mathrm{C}$ module placed behind. The $\mathrm{C}$ module (Table. 1) is a non compensating ( $\mathrm{e} / \pi \sim 1.4$ ) hadron calorimeter with a hadronic energy resolution of $\sim 100 \% / \sqrt{ } \mathrm{E}$. The lateral energy leakage is estimated from recent measurements with a three module assembly to be less than $5 \%$. However, we have not applied any leakage corrections to the results which follow. The pion showers are thus longitudinally sampled with three different sampling fractions. The total reconstructed pion energy is taken to be the sum of energies seen in all the samplings of six towers and the two samplings of the C-Module i.e.

$\mathrm{E}_{\text {rec }}(\mathrm{GeV})=\sum_{\mathrm{T}=1,6}\left\{\alpha \sum_{\mathrm{i}=1,4} \mathrm{Q}_{(\mathrm{i}}(\mathrm{fC})+\beta \sum_{\mathrm{i}=5,6} \mathrm{Q}_{\mathrm{i}}(\mathrm{fC})\right\}+\beta_{\mathrm{c}} \sum_{\mathrm{i}=1,2} \mathrm{E}_{\mathrm{i}}^{\mathrm{c}}$

where $\alpha$ is fixed to the value found earlier for electrons and $\beta$ and $\beta_{c}$, also assumed to be energy independent calibration factors, are determined from the minimisation of the difference between the reconstructed and the beam energies. $\alpha$, determined from the electron runs, is 0.032 $\mathrm{GeV} / \mathrm{fC}$ and $\beta$ is found to be $0.083 \mathrm{GeV} / \mathrm{fC}$. $\beta_{\mathrm{C}}$ is found to be 1.452 . After subtracting the noise contribution, amounting to $940 \mathrm{MeV}$, we obtain an energy resolution (Fig.2) given by $\sigma(\mathrm{E}) / \mathrm{E}=(0.581 \pm 0.009) / \sqrt{ } \mathrm{E} \oplus(0.068 \pm 0.002)$.

The relatively large constant term is due to the non-uniform sampling of the hadronic showers. The pulse height distribution of $50 \mathrm{GeV}$ pions is shown in Fig 3 and can be seen to have negligible tails.

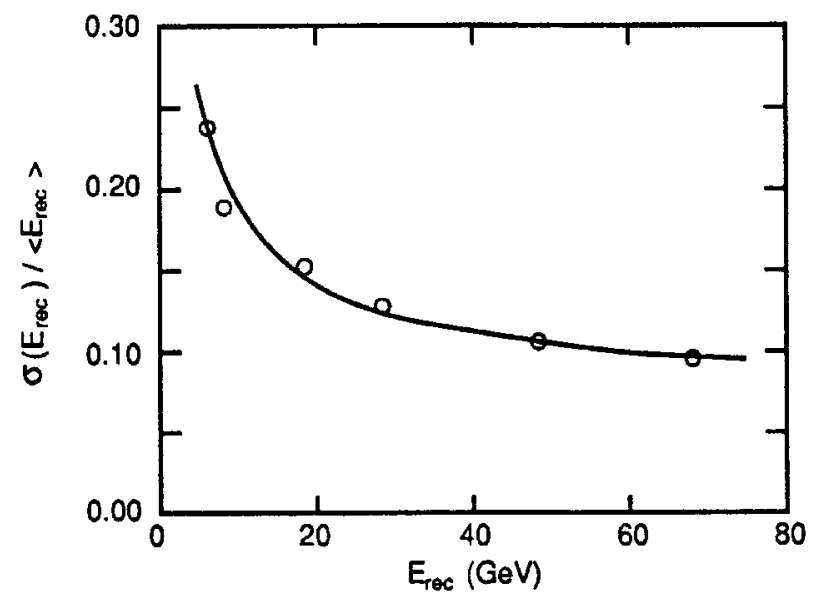

Fig.2 The energy resolution for pions as a function of energy for the $S G+C$ modules.

\section{The Electron / Pion Response.}

Fig. 4 illustrates, as a function of the particle energy, the ratio of the reconstructed electron and pion energies taken from the mean of a Gaussian fitted to the measured energy distributions. The e/ $\pi$ ratio varies between 1.08 at 7 $\mathrm{GeV}$ to 1.02 at $70 \mathrm{GeV}$. It can be seen that a $2.35 \lambda \mathrm{SG}$ U/TMP module backed by the $\mathrm{C}$ module results in a configuration that is compensating.

\section{The Uniformity of Response}

A beam of $70 \mathrm{GeV}$ electrons was fired at the centres of all towers of a SG module to study the uniformity of the response. After correcting the pulse 


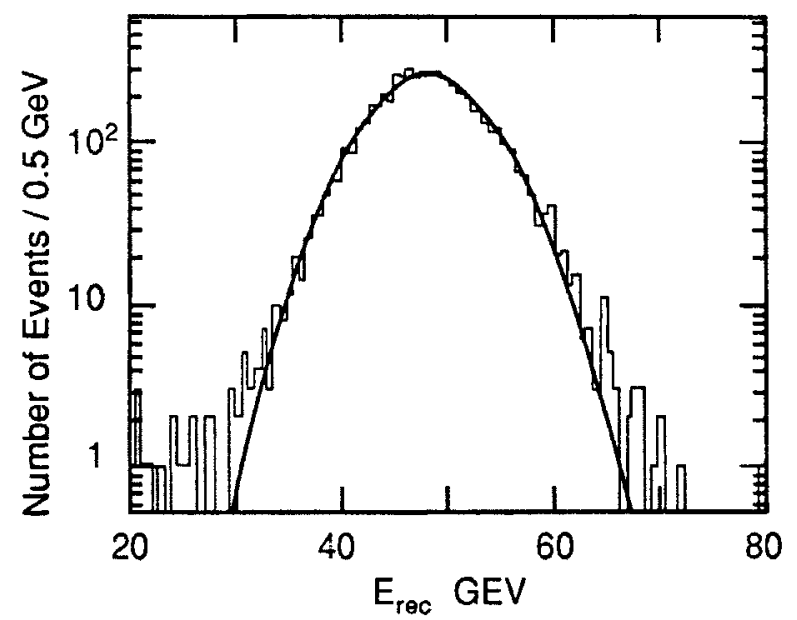

Fig.3 The reconstructed pion energy distribution in SG module.

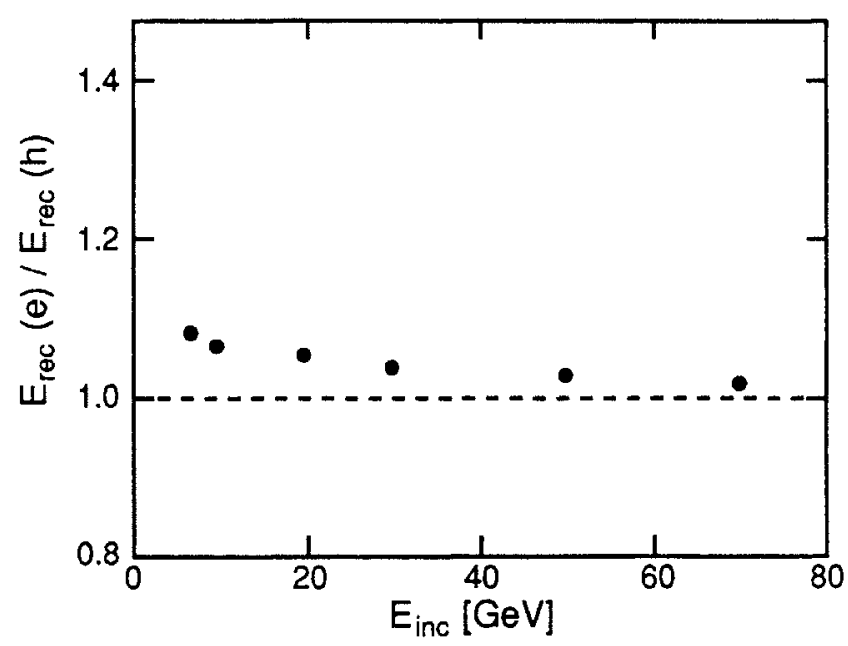

Fig.4 The ratio of the reconstructed electron to pion energies as a function of the incident particle energy for $\mathrm{SG}+\mathrm{C}$ modules.

height for any disconnected electrodes we find that the responses show an r.m.s. variation of $0.7 \%$ (Fig. 5a).

A more detailed scan over areas with cracks or steel bars was also carried out with a $40 \mathrm{GeV}$ electron beam. The position of each incident electron was measured with a proportional wire chamber situated just in front of the module. The results (Fig. 5b) show clearly the decrease in the collected charge when the electrons traverse the stainless steel frames of the chambers. The decrease is largest where the two adjacent chambers meet. The small increase in the collected charge occurs when the beam is centred on a gap between adjacent electrodes eg. T5 \& T7.

\section{The Influence of the Electric Field.}

The total number of ion pairs produced in liquid TMP per $100 \mathrm{eV}$ is almost the same as in the gas phase. However not all ionization electrons become "free". The

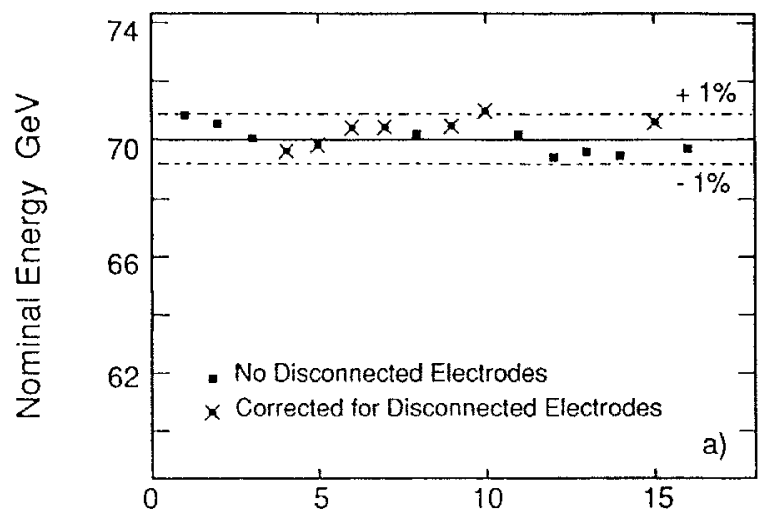

Number of Tower
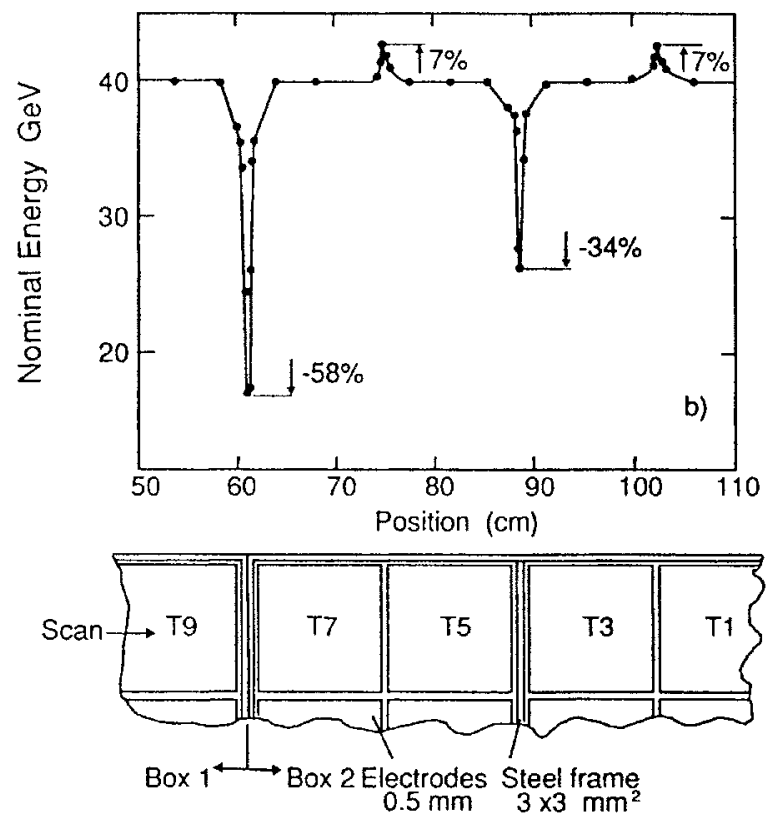

Fig.5 The uniformity of response a) to $70 \mathrm{GeV}$ electrons incident in the centre of towers of a SG module b ) to $40 \mathrm{GeV}$ electrons incident over areas containing cracks and steel frames.

motion of these electrons in the liquid is governed by their diffusion in the liquid and by the Coulomb attraction of the positive ions. A certain number of the electrons will recombine with the positive ions. The escape probability for an electron from its parent ion has been calculated by Onsager [6]. It is modified by an externally applied electric field. In the approximation of a low externally applied electric field and a low density of ionization, eg. that created by a minimum ionizing particle (mip), where only the recombination between an electron and its parent ion is important, the free ion yield and hence the observed charge is given by

$\mathrm{G}_{\mathrm{fi}}=(100 / \mathrm{W}) \exp \left(-\mathrm{r}_{\mathrm{c}} / \mathrm{r}\right)[1+\alpha \mathrm{E}]$

where $r_{c}$ is the Onsager escape radius and $\alpha=$ $\mathrm{e}^{3} /\left(8 \pi \varepsilon_{0} \varepsilon \mathrm{k}^{2} \mathrm{~T}^{2}\right)$. However for highly ionizing particles a 


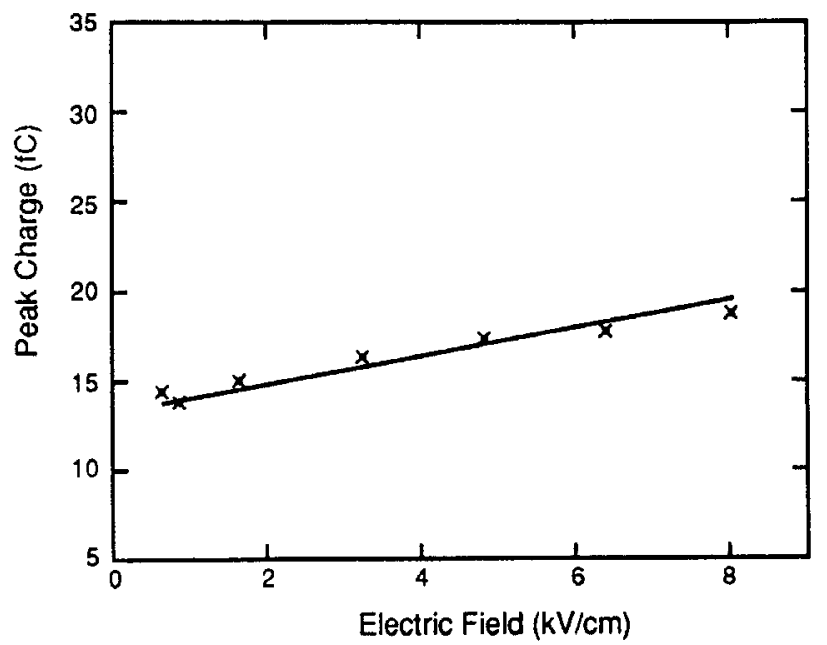

Fig.6 The response of SG module to $70 \mathrm{GeV}$ muons as a function of the electric field.

dense column of electron-ion pairs is created and one has to consider volume (or columnar) recombination in addition. An electron in the column can recombine with an ion other than the parent one. In this case $\mathrm{G}_{\mathrm{fi}}$ will be smaller and the field dependence will be different.

As the electron showers consist mainly of mip's whereas pion showers contain densely ionizing particles, especially in hydrogeneous media like TMP, the field dependence of the collected charge for electrons and muons should be different from that for pions. Fig.6 shows the response of a SG module to $70 \mathrm{GeV}$ muons as a function of the field. The slope of the line shown in the graph is a prediction of the linear approximation of Onsager's theory with $\alpha=0.057 \mathrm{~cm} / \mathrm{kV}$. Electron showers have a similar dependence on the field as evident in Fig. 7 for electrons of an energy of $40 \mathrm{GeV}$ in the FW module. The different field dependence for pion showers can be seen by examining the ratio of the collected charge $Q_{\mathrm{e}} / \mathrm{Q}_{\pi}$ for the VFW module shown in Fig. 8. A clear decrease of this ratio with increasing electric field is seen. Such a strong dependence is not observed in the SG module.

The pion energy resolution in SG module [4] improves significantly with higher electric field whereas the electron energy resolution does not change above a relatively low electric field of $\sim 2 \mathrm{kV} / \mathrm{cm}$, as observed previously in the VFW module [2]. The improvement in pion energy resolution is due to the different electric field dependence of the collected charge for heavily ionizing particles, as opposed to minimum ionizing ones.

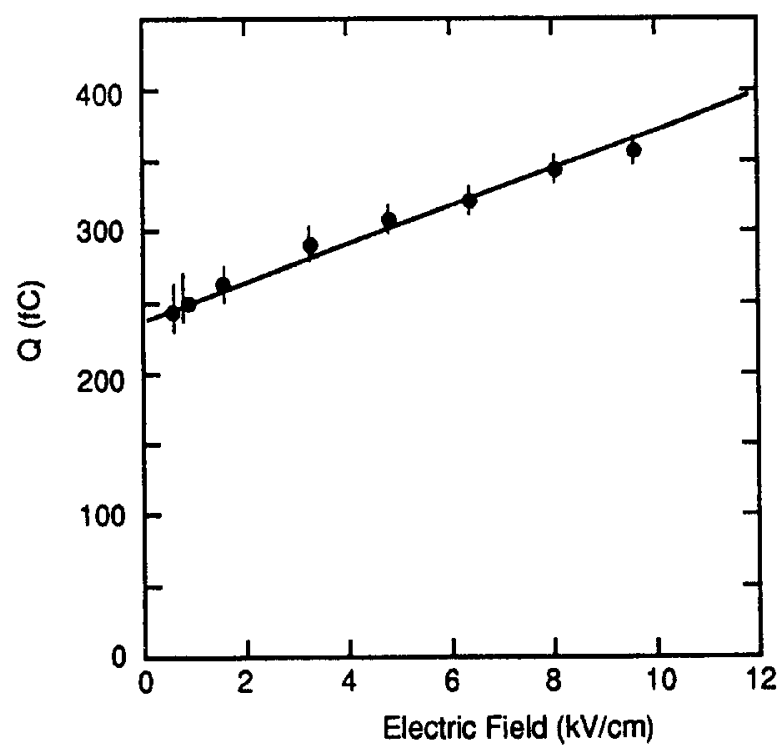

Fig.7 The response of the FW module to $40 \mathrm{GeV}$ electrons as a function of the electric field.

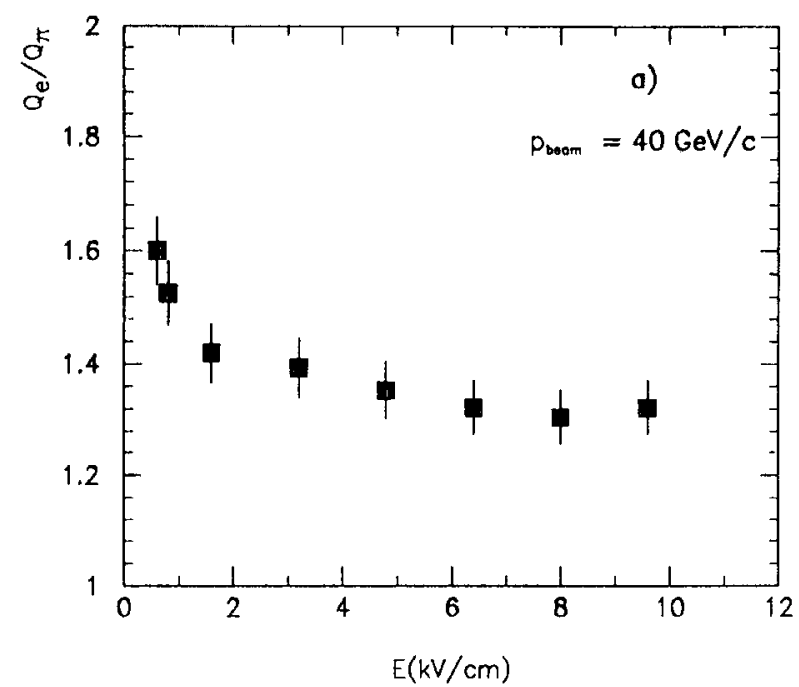

Fig.8 The response of the VFW module to $40 \mathrm{GeV}$ pions as a function of the electric field.

\section{The Postion Detector.}

We have determined the position resolution for single electromagnetic showers by comparing the position of each incident electron, measured by the wire chamber placed directly in front of the calorimeter module, with that evaluated by the method of weighted mean (weighted by the pulse height). The resulting distribution of the difference in the two position measurements as a function of the shower energy is shown in Fig.9. The spatial resolution is $\leq 1 \mathrm{~mm}$ at high energies.

\section{The Electron - Pion Separation.}

The difference between the energy deposited by electrons and by pions in the lateral and longitudinal 


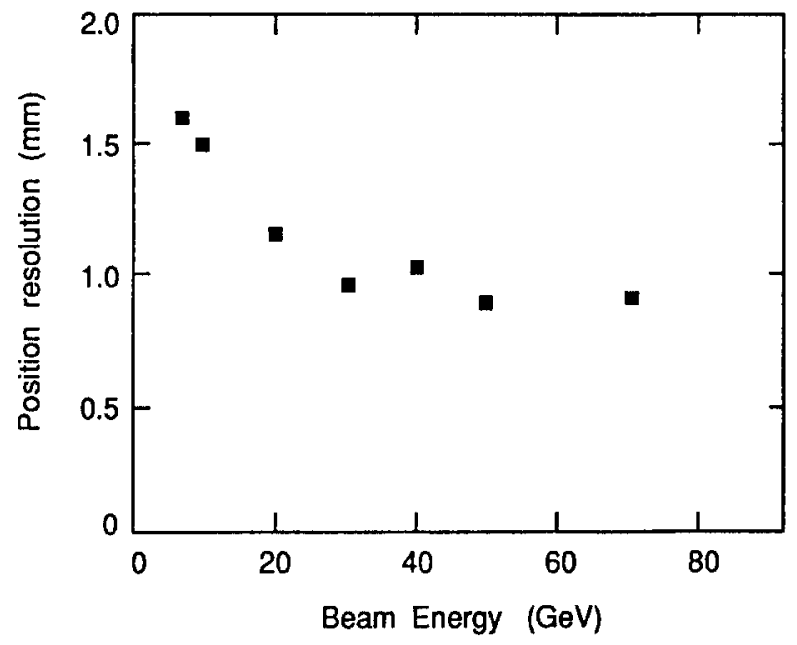

Fig.9 The spatial resolution in the position detector for single electromagnetic showers as a function of incident energy.

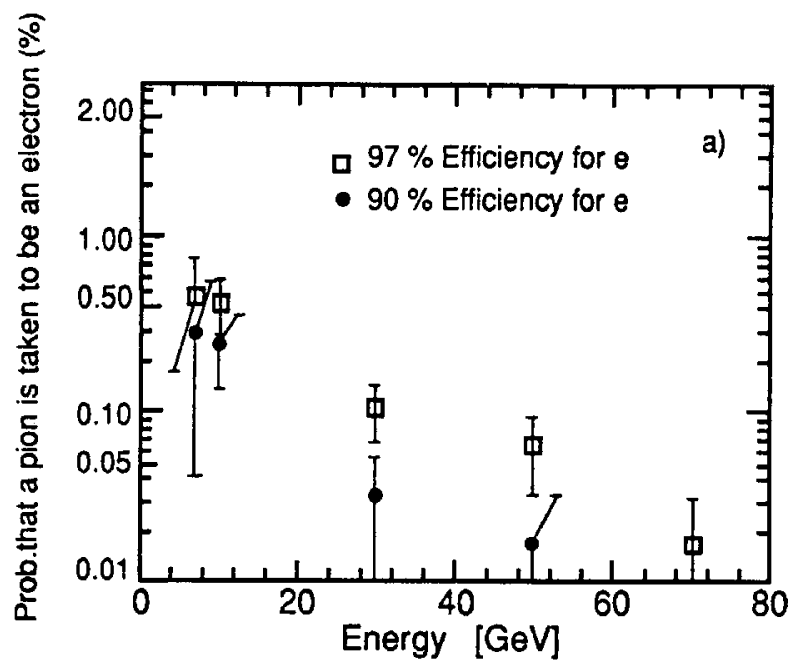

Fig.10 The probability as a function of energy that a pion looks like an electron.

semplings and in the position detector can be used to distinguish between them. We have studied [4] the e / $\pi$ separation in a SG module for single particles with an incident energy ranging from $7 \mathrm{GeV}$ to $70 \mathrm{GeV}$. The probability that a pion looks like an electron is shown in Fig. 10 for electron identification efficiencies of $90 \%$ and $97 \%$. The indicated error bars are purely statistical.

We have also determined the electron - pion separation capability of the PD when used independently. The electron identification efficiency is defined by a cut on the electron pulse height distribution. The same cut is then applied to the pion pulse height distribution and the resulting probability that a pion passes the electron cut is shown in Fg. 11 for electron identification efficiency of $90 \%$.

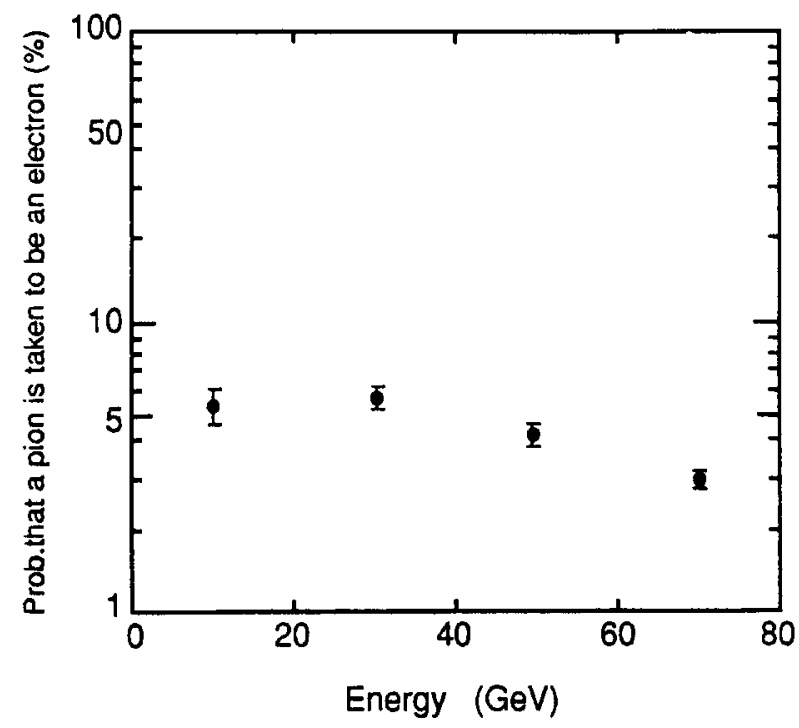

Fig.11 The probability, as a function of energy, that a pion passes the electron cuts in the PD for an electron efficiency of $90 \%$.

\section{Acknowledgements}

I would like to thank my colleagues in UAl who have worked on the development, construction and tests of the various U/TMP calorimeter modules.

\section{References}

[1] M. Albrow et al., NIM A265 (1988) 303.

[2] C. Bacci et al., NIM A292 (1990) 113.

[3] C. Bacci et al., Submitted to NIM

[4] R. Apsimon et al., To be submitted to NIM.

[5] K. Ankoviak et al., NIM A279 (1989) 83.

[6] L. Onsager, Phys.Rev. 54 (1938) 554. 